\title{
Le malade partenaire de la recherche et des soins : paradoxe ou nécessité?
}

Le malade peut-il être partenaire du médecin dans le cadre de la recherche clinique, et /ou dans la mise en place d'un projet d'établissement? Les premiers États généraux du cancer, organisés par la Ligue nationale contre le cancer ont eu lieu à Paris en novembre 1998. À la suite de cet événement singulier, deux expériences inédites ont été menées.

\section{8 - À Paris}

Persuadés qu'une complémentarité médecin/malade est devenue nécessaire, voire impérative, le Dr F. May-Levin, médecin conseil au siège de la Ligue nationale contre le cancer et le Pr D. Maraninchi, alors directeur de l'Institut Paoli-Calmettes (IPC) à Marseille, proposent de mettre en place un comité de patients pour la recherche clinique en cancérologie (CPPRCC). Projet audacieux ! Qui défie résolument les rapports médecin/malade, établis depuis... Hippocrate.

La finalité du projet était de faire du patient un partenaire actif dès lors qu'il entre dans un essai clinique, afin d'associer sa propre connaissance de la maladie au savoir médical, cela en vue d'améliorer sa qualité de vie dans le cadre des contraintes imposées par le protocole. La charte établie précise la relation tripartite liant la FNCLCC (fédération nationale des centres de lutte contre le cancer), la Ligue nationale contre le cancer et un groupe de huit malades sélectionnés par la Ligue.

Les objectifs, travaillés ensemble, sont volontairement peu nombreux. Ils consistent:

1. à relire les protocoles d'études cliniques et la lettre d'information qui les accompagne, celle-ci devant être signée par les malades avant d'entrer dans l'essai ;

2. à proposer des améliorations afin que cette lettre soit claire, loyale, explicite et précise en ce qui concerne les objectifs, les moyens, les contraintes, et les effets secondaires inhérents à l'étude ;

3. à valider un plan de traitement, de surveillance, de suivi, simple et schématique, accessible à tous, afin d'assurer une visibilité globale des impératifs, pour permettre une organisation personnelle quotidienne.

Quelle témérité de la part de nos mentors ! Nous les malades étions persuadés que jamais des promoteurs n'accepteraient une telle association ! Comment imaginer être assis autour d'une même table, et argumenter une partie de leur travail. Et pourtant... d'année en année, 21 promoteurs nous ont fait confiance. En 2004, une fédération de comités de patients voit le jour. Le nombre de relecteurs est passé de 8 à 40 , répartis sur toute la France.

Actuellement plus de 300 protocoles et lettres destinés aux patients ont été relus. Environ 800 suggestions ont été retenues et validées.

\section{2 - À Marseille}

À l'instigation du Pr Maraninchi et de son équipe, I'IPC organise en son sein la mise en place d'un comité de patients. Sa finalité est d'améliorer les conditions d'accueil, d'information, de séjour de tout consultant hospitalisé ou suivi à l'Institut. Une charte établit les règles d'un partenariat bipartite entre l'IPC, qui s'engage à fournir au comité de patients les cadres d'organisation et de fonctionnement liés à ses missions, et le comité de 8 à 10 personnes qui s'engage à respecter les modalités de fonctionnement fixées par l'Institut.

Là encore, les objectifs sont volontairement peu nombreux. Ils établissent les priorités suivantes:

1. l'information du patient doit être loyale, complète, intelligible, sur le déroulement de sa prise en charge ;

2. I'identification et l'amélioration des motifs d'insatisfaction du malade/de ses proches ;

3. la participation avec les professionnels de l'IPC à la réflexion concernant les grands projets institutionnels, en cours ou à venir.

À ce jour, les actions du comité de patients ont été relativement nombreuses. Par exemple : la participation à la mise en place d'une consultation systématique auprès d'une infirmière après la consultation médicale; la participation à la création d'un espace rencontre/information (ERI) ouvert à tous, malades/familles/visiteurs; des dialogues avec les professionnels lors de leurs réunions concernant la certification de l'établissement (2010); une participation régulière aux réunions de travail relatives à la création des nouveaux bâtiments (IPC II, IPC III).

Et même !... une participation au projet médical, voulue par le Pr Viens, directeur de l'IPC, en juillet 2011 [1].

Ainsi, à la question : le malade peut-il être un partenaire dans la recherche clinique, dans la mise en place d'un projet d'établissement, dans l'élaboration du projet médical ? Démonstration est faite que la réponse est oui.

Le malade doit-il être partenaire du médecin? Dans le plan cancer II, I'INCa souhaite dynamiser la recherche clinique en prenant « l'avis des comités de patients... en articulation avec la consultation des comités de protection des personnes (CPP)» [2]. La réponse est : certainement. Alors... soyons attentifs.

The patient partner of research and care:paradox or necessity?

\section{RéF́́RENCES}

1. Viens P. Nouveau projet-nouveaux patients-nouvelle dimension. IPC Info, 2011.

2. Plan cancer 2009/2013. Mesure IV, page 27. Paris : Éditions INCa, 2009. http://www.plan-cancer.gouv.fr

Sylvia Achin pour le Comité de Patients IPC et la Fédération des Comités de Patients FNCLCC et Ligue Nationale Contre le Cancer 


\section{> Grâce à $m / s$, vivez en direct les progrès des sciences biologiques et médicales}

\section{médecine/sciences}

\section{Chaque mois,} avec les articles de référence de $\mathrm{m} / \mathrm{s}$

Chaque jour, sur www.medecinesciences.org

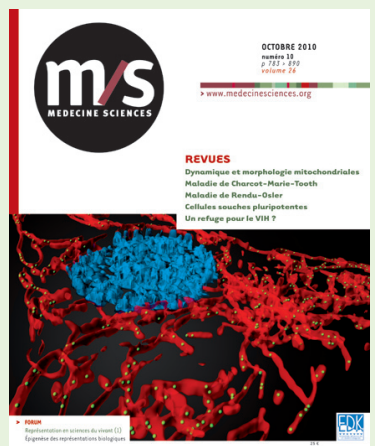
médecine/sciences est indexé dans

\author{
PubMed/Medline \\ Current Contents, série Life Sciences \\ EMBASE/Excerpta Medica \\ PASCAL \\ CABS \\ BIOSIS
}

> Des articles rédigés par des médecins et des chercheurs reconnus sur la scène internationale qui posent avec rigueur les bases des débats scientifiques.

> Des synthèses, éditoriaux, dossiers techniques et analyses toujours replacés dans leur contexte pour que l'information soit la plus exacte, intelligible et objective.

> La dimension humaine privilégiée, avec l'analyse des retombées diagnostiques, thérapeutiques, la prévention et l'éthique liées aux nouvelles avancées.

> Un panorama clair et concis de l'actualité scientifique: des nouvelles, des brèves, des données chiffrées, des repères et perspectives pour qu'aucun fait significatif ne vous échappe.

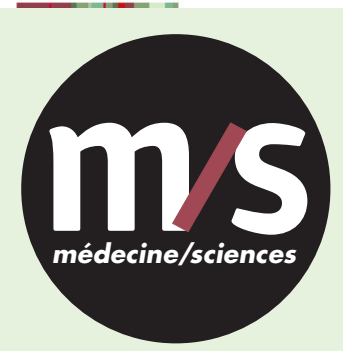

Tarifs d'abonnement $\mathrm{m} / \mathrm{s}$ - 2012 Mensuel - 10 numéros/an

\section{Abonnez-vous}

\section{à médecine/sciences}

Je souhaite m'abonner à $\mathrm{m} / \mathrm{s}$ :

Nom:

\section{Adresse}

Code postal

Pays :

$\varepsilon$-mail-obligatoire

Je choisis l'abonnement:

\begin{tabular}{cc}
\multicolumn{2}{c}{ Particuliers } \\
Papier & Électronique \\
+ & seul \\
Électronique &
\end{tabular}

$\begin{array}{lllll}\text { France } & \square 205 € & \square 140 € & \square 461 € & \square \text { Surdevis } \\ \text { UE } & \square 272 € & \square 140 € & \square 562 € & \square \text { Surdevis } \\ \text { Reste du monde } & \square 272 € & \square 117 € & \square 583 € & \square \text { Surdevis }\end{array}$

$\times$ Jondeun ussificas

* Joindre un justificatif
Prénom :

Ville :

\begin{tabular}{|c|c|}
\hline \multicolumn{2}{|c|}{ Institutions } \\
\hline $\begin{array}{c}\text { Papier } \\
+ \\
\text { Électronique }\end{array}$ & $\begin{array}{c}\text { Électronique } \\
\text { seul }\end{array}$ \\
\hline$\square 461 €$ & $\square$ Sur devis \\
\hline$\square 562 €$ & $\square$ Sur devis \\
\hline$\square 583 €$ & $\square$ Sur devis \\
\hline
\end{tabular}

\author{
Mon règlement : \\ $\square$ Parmail edk@edk.fr \\ Uniquement pour les paiements par carte bancaire \\ $\square$ Par fax en envoyant ce bulletin au 0143293262 \\ Uniquement pour les paiements par carte bancaire

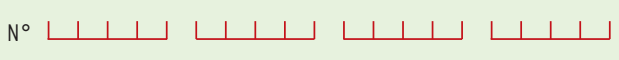 \\ Date d'expiration $\downarrow \downarrow \downarrow \downarrow \downarrow$ Signature: \\ $\mathrm{N}^{\circ}$ de contrôle au dos de la carte $\llcorner\perp \perp$
$\square$ Par chèque à l'ordre de médecine/sciences, en envoyant ce bulletin à :
Éditions EDK - Groupe EDP sciences
17, avenue du Hoggar - P.A. de Courtabœuf
91944 Les Ulis Cedex A, France

Pour recevoir une facture, cochez cette case $\square$ 


\section{Notes}
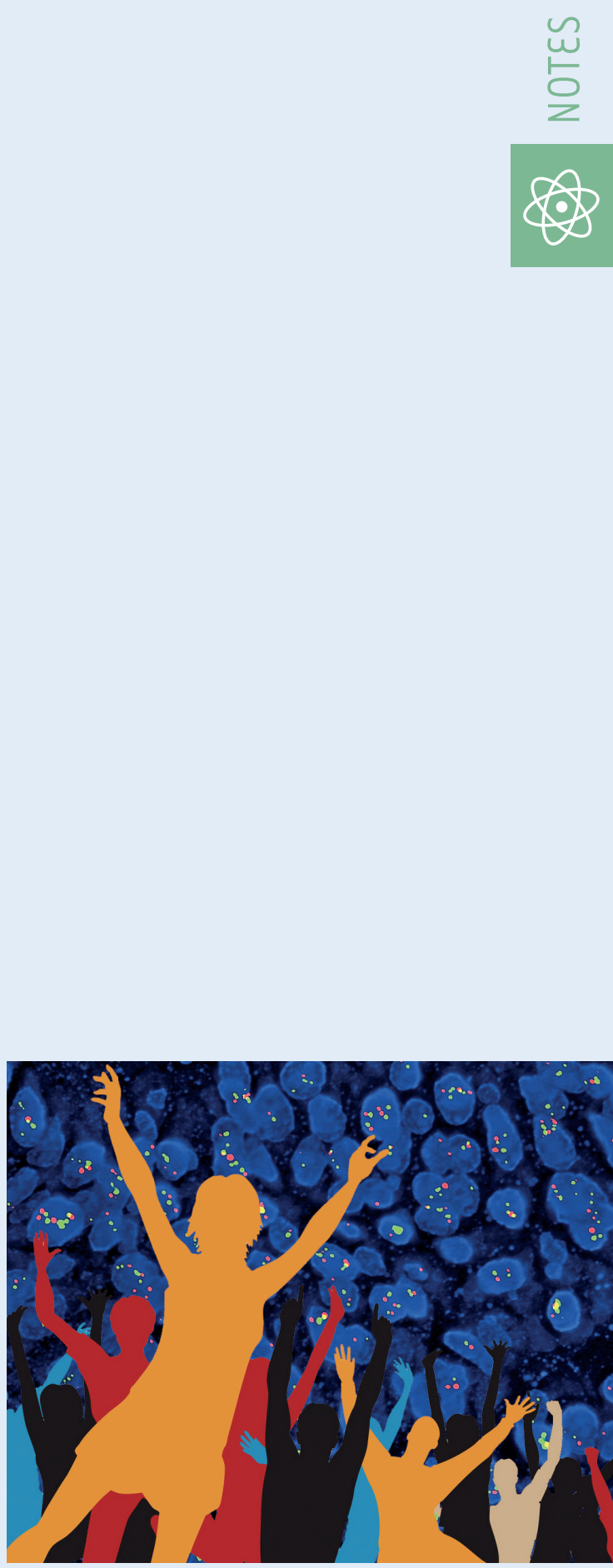
